\title{
Erratum to: Introduction of an examination and treatment for Helicobacter pylori infection in high school health screening
}

\author{
Taiji Akamatsu $\cdot$ Shinya Ichikawa $\cdot$ Sadahide Okudaira \\ Shuichi Yokosawa $\cdot$ Yugo Iwaya · Tomoaki Suga • \\ Hiroyoshi Ota $\cdot$ Eiji Tanaka
}

Published online: 7 September 2012

(c) Springer 2012

\section{Erratum to: J Gastroenterol (2011) 46:1353-1360 DOI 10.1007/s00535-011-0450-6}

Errors appeared in the above-cited article. Corrections are shown below.

In the Abstract (p. 1353), in the "Methods" subsection the second sentence should read as follows:

They were first examined with a urinary rapid test kit based on immunochromatographic technology for detection of the antibody to H. pylori (RAPIRAN ${ }^{\circledR}$ ).

In the Methods section (p. 1354), in the subsection headed "Screening examination of $H$. pylori infection at the high school," the first sentence should read as follows:

Students were first examined using a urinary rapid test kit based on immunochromatographic technology for detection of the antibody to $H$. pylori (RAPIRAN ${ }^{\circledR}$ : Otsuka Pharmaceuticals, Tokyo, Japan).

The online version of the original article can be found under doi:10.1007/s00535-011-0450-6.

T. Akamatsu ( $₫)$

Endoscopy Center, Suzaka Hospital, Nagano Prefectural

Hospital Organization, 1332 Suzaka,

Suzaka, Nagano 382-0091, Japan

e-mail: akamatsut@suzakahp.jp

T. Akamatsu $\cdot$ S. Ichikawa $\cdot$ S. Yokosawa $\cdot$ Y. Iwaya

T. Suga $\cdot$ E. Tanaka

Department of Internal Medicine, Gastroenterology,

Shinshu University School of Medicine, Matsumoto, Japan

S. Okudaira

Okudaira Clinic, Matsumoto, Japan

H. Ota

Department of Biomedical Sciences, School of Health Sciences,

Shinshu University School of Medicine, Matsumoto, Japan
In the Discussion section (p. 1357, left column, lines 9-16), the sentence beginning "Kato et al." should read:

Fujisawa et al. [12] reported that the accuracy of a urinary rapid test kit based on immunochromatographic technology for detection of the antibody to $H$. pylori was almost equal to the accuracy of serum-based enzymelinked immunosorbent assay. For these reasons, we think that the screening method using a urinary rapid test kit based on immunochromatographic technology for detection of the antibody to $H$. pylori is an appropriate way to screen for $H$. pylori infection as part of the school health screening.

In the Reference section (p. 1360), entry \#12 should read as follows:

Fujisawa T, Kaneko T, Kumagai T, Akamatsu T, Katsuyama T, Kiyosawa K, et al. Evaluation of urinary rapid test for Helicobacter pylori in general practice. J Clin Lab Anal. 2001;15:154-9. 